\title{
EXISTIERT EIN INTERNATIONALES AMERIKANISCHES OOFFENTLICHES RECHT?
}

\author{
Von Antonio Salum Flecha
}

Das internationale Recht war ursprünglich nichts anderes als europäisches Recht, das ist die herrschende Meinung der Völkerrechtler, die sich darauf berufen, daß das „Droit Public de l'Europe“ lange Zeit seine einzige Rechtsgrundlage war. Erstmals im Jahre 1776 wurden die Grenzen dieses europäischen internationalen Rechtes gesprengt; das war, als die Vereinigten Staaten von Amerika sich anschlossen, indem sie feierlich ihre Unabhängigkeit proklamierten und ihre Grundrechte verkündeten. Diesem bedeutenden Ereignis folgte nach und nach die Emanzipation der lateinamerikanischen Nationen, die nun innerhalb des internationalen Rechtes den Platz einnehmen konnten, der sie befähigte, neue und entscheidende Beiträge zu liefern, die - im Rahmen einer stetigen Verbesserung der juristischen Voraussetzungen $-\mathrm{zu}$ dem wurden, was viele eminente Völkerrechtler das Internationale Offentliche Recht Amerikas nennen.

Chronologisch folgte die Proklamation der Monroe-Doktrin, die mit unerschütterlichem Glauben von den USA verfochten wurde, so daß es ihr gelang, den amerikanischen Kontinent jeder weiteren europäischen Vormundschaft zu entziehen. Ohne diese Doktrin hier analysieren zu wollen, die auf die verschiedenste Weise interpretiert wurde, muß anerkannt werden, daß sie der Anfang der Ausweitung des Internationalen Offentlichen Rechtes ist.

Auf diese Weise verwandelte sich unsere Wissenschaft in ein Internationales Recht der Staaten christlicher Zivilisation, bis die Türkei im Jahre 1856 dem Konzert der europäischen Staaten beitrat und sich später Staaten wie Japan, China und andere der asiatischen und afrikanischen Kontinente der internationalen Gemeinschaft anschlossen. Damals wurde die ideale, höchste und letzte Universalität des Internationalen Rechtes erreicht, ein Prozeß, der sich durch die zwei Weltkriege beachtlich schnell entwickelte.

Am 28. Juli 1919 wurde in Versailles der historische Vertrag unterzeichnet, der dem Ersten Weltkrieg ein Ende setzte. Kraft eines Abkommens, das in diesen Vertrag einbezogen wurde, wurde der Völkerbund als Folge des letzten der vierzehn Punkte von Woodrow Wilson gegründet, der am 20. Januar 1920 seine legalen Funktionen aufnahm. Innerhalb des Bundes existierten verschiedene Institutionen mit internationalem Charakter, wie die Internationale Arbeitsorganisation und der Ständige Internationale Gerichtshof in Den Haag, der sich im Jahre 1922 konstituierte.

Jedoch der Bund oder diese Liga der Nationen, deren Mitglieder nur 29 Länder zählten, war weit davon entfernt, einen universalen Charakter zu haben, der für die Erfordernisse der internationalen Politik der Großmächte unerläßlich gewesen wäre. Hinzu kamen weitere organisatorische und funktionelle Unzulänglichkeiten, und so war es konsequent, daß die Liga bei Ausbruch des Zweiten Weltkrieges im Jahre 1939 auseinanderfiel, worauf zur Mitte des darauffolgenden Jahres der Beschluß gefaßt wurde, die Funktionen zu dezentralisieren. Es interessiert in diesem Zusammenhang nicht, die Fehler dieser ersten Weltorganisation aufzuzählen, vielmehr möchte ich den chronologischen Verlauf der Ereignisse darlegen. Trotzdem muß erwähnt werden, daß diese erste Initiative die absolute Staatssouveränität, die im 19. Jahrhundert dogmatisch und praktisch feststand, zu beschränken begann und so eine wertvolle Erfahrung für spätere Versuche darstellt.

Der Zweite Weltkrieg, der als Folge irriger Dispositionen des Versailler Vertrages und des Entstehens neuer aggressiver und machtlüsterner Regierungsformen in Europa ausbrach, macht, um die Worte des sowjetischen Koronin zu gebrauchen, die Existenz eines „Internationalen Übergangsrechtes“ unmöglich. 
Bei Ende des Zweiten Weltkrieges, 1945, schufen die alliierten Siegermächte einen außerordentlichen Präzendenzfall, indem sie einen internationalen Gerichtshof in Nürnberg bildeten, um über die sogenannten „großen Kriegsverbrecher" zu Gericht $\mathrm{zu}$ sitzen, wodurch sie vielleicht die Grundlagen für ein neues Weltstrafrecht gesetzt haben; dies könnte der Anfang einer neuen Ära des Internationalen Offentlichen Rechtes sein.

Alsbald bildete sich die Organisation der Vereinten Nationen (UNO) und trat die Nachfolge des erloschenen Völkerbundes an. Ihre Konstitution wurde am 26. Juni 1945, kurz vor Beendigung der Feindseligkeiten, in San Franzisco (Kalifornien) unterzeichnet. Die einzig mögliche Erklärung für diese Gründung - soviel man auch darüber diskutiert haben mag - ist darin zu sehen, daß es die Großen Fünf Mächte gab, die sich ein Vetorecht und den ständigen Sitz im Sicherheitsrat erwirkten.

So entstand diese Organisation im Durcheinander der damals bestehenden Leidenschaften, aber es bestand kein genauer Plan für die Maßnahmen, die nach Beendigung der Kriegsereignisse gefaßt werden mußten. Daher entstand das Problem, daß Nationalchina im Sicherheitsrat vertreten war, während Staaten wie Deutschland und bis vor kurzem Japan, Spanien usw. fehlten.

Schließlich müssen wir darauf hinweisen, daß das hervortretende Merkmal der jüngsten Zeit die Tendenz der Regierungen ist, zu einem Zusammenschluß der Staaten zu gelangen und regionale Systeme dieses Mal der Struktur der Charta einzuverleiben.

\section{Das Internationale Öffentliche Amerikanische Recht}

Für uns Lateinamerikaner ist die europäische Doktrin nicht mehr Grundlage des Internationalen Offentlichen Amerikanischen Rechts, denn dieses hat sich historisch und juristisch etabliert.

Beginnen wir mit der Erklärung, daß wir zwar Anhänger der Naturrechtslehre sind, was uns aber keineswegs daran hindert, partikulare internationale Rechtsformen anzuerkennen. Deshalb haben war ja auch bereits vom Internationalen Europäischen Recht, vom Recht der christlichen Staaten usw. gesprochen, also von Rechtsformen relativer Art, die erst im Laufe des Jahrhunderts erweitert wurden.

Wir stimmen auch mit Scelle überein, wenn er sagt: „Den verschiedenen internationalen Teilgesellschaften entsprechen eigene angemessene Ordnungen. Tatsächlich bildeten sich im Bereich der universellen oder ökumenischen internationalen Gesellschaft - und sogar vor deren Bestehen - Gruppierungen von Völkern oder Staaten. Diese werden zusammengeführt durch Beziehungen, die um so fester sind, als sie auf gemeinsamer Abkunft oder Rasse oder geographischer Verbundenheit beruhen, und die vor allem vom Volumen des gegenseitigen Handels und monetären Austausches bestimmt werden und so in Europa und Amerika Verhältnisse schaffen, die regional besonders harmonisch sind und doch im Rahmen der Ordnung des globalen internationalen Rechtes bleiben."

Wir stimmen dieser Stellungnahme zu, müssen aber dabei daran erinnern, daß bestimmte Probleme des Internationalen Rechtes in Amerika spezielle Lösungen fanden, die oftmals anders waren als die Prinzipien der Alten Welt und ihnen sogar gelegentlich widersprachen.

Wir teilen also das Internationale Recht ein in Universales oder Ókumenisches, in Allgemeines und Partikulares. Wenn wir nun vom internationalen europäischen oder amerikanischen Recht sprechen, dann ist es logisch, daß wir es in die zweite Gruppe stellen, wonach es gleichzeitig allgemein und partikular ist. Das partikulare Recht kann kontinental oder regional sein, was aus folgender Skizze ersichtbar ist: 


\begin{tabular}{l|l}
$\begin{array}{l}\text { Internationales } \\
\text { Recht }\end{array}$ & Allgemein
\end{tabular}$\left\{\begin{array}{l}\text { Kontinental (amerikanisch oder europäisch) } \\
\text { Regional (regionale Zusammenschlüsse) } \\
\text { Partikular }\end{array}\left\{\begin{array}{l}\text { Einzelne Vereinbarungen } \\
\text { zwischen verschiedenen Staaten, } \\
\text { z. B. ein bilateraler Handelsvertrag }\end{array}\right.\right.$

Mit dieser Einteilung schließen wir uns denen an, die die Geltung eines juristischen Zweiges des speziellen Völkerrechts der amerikanischen Nationen vertreten.

Diese Urteilselemente erlauben uns, folgende Definition des Internationalen Offentlichen Amerikanischen Rechtes zu formulieren: Das Zusammentreffen normativer Prinzipien und juristischer Institutionen, die von der Rechtslehre und den internationalen Praktiken getragen und historisch und politisch untermauert sind, sind den amerikanischen Nationen eigen.

In unserem Fall kommt das Zusammentreffen nur solcher normativer Prinzipien in Frage, die einen offenkundigen Einfluß auf die amerikanische Rechtsordnung nehmen und uns daher gestatten, das Recht ganz speziell anzuwenden. Die fundamentale historische Norm zur Begründung der Grenzziehung, das „uti possidetis iuris", legt die Unverletzlichkeit der staatlichen Ordnung fest; es gilt nur in der Neuen Welt und ist ein Beispiel für das, was wir dargelegt haben. Vielleicht könnte man in die juristischen Institutionen auch das Asylrecht einbeziehen (es ist typisch iberoamerikanisch, da die Vereinigten Staaten es nicht anerkennen), denn obwohl es nicht ein eigentlicher Bestandteil des Internationalen Rechtes ist, gehört es doch seinem Wesen nach dazu.

Wenn man von der Doktrin des Panamerikanismus, genau gesagt, vom Internationalen Offentlichen Amerikanischen Recht spricht, so ist das nicht nur ein Aufzählen berühmter Völkerrechtler des Kontinents, sondern eine verdiente und tiefempfundene Huldigung für alle diejenigen, die mit ihren Ideen dazu beigetragen haben, die mächtige Pyramide zu errichten, die der entscheidende Abschnitt in der Geschichte des Universalen Internationalen Rechtes ist. Darum soll unsere Huldigung ihnen allen in der Person des einen großen chilenischen Völkerrechtlers gelten, Alejandro Alvarez, der eine bedeutende These zu dieser Materie der Offentlichkeit schenkte. $\mathrm{Da}$ wir schon berühmte Völkerrechtler nennen, dürfen wir Namen wie Juan Bautista Alberdi nicht übergehen, von dem der Argentinier César Díaz Cisneros sagte, er sei der wahre Begründer des Internationalen Amerikanischen Rechtes, das er als Organismus der Lehren oder Prinzipien, die Gemeingut des Kontinents seien, aufgefaßt habe. Wir denken auch an Professor Lucio Moreno Quintana und an einen neuen Autor, Juan Carlos Puig, die sich in brillanter Weise für das Recht eingesetzt haben, das wir vertreten.

Die internationalen Praktiken, auf die unsere Definition anspielt, durchziehen die Reihe der interamerikanischen Konferenzen und Kongresse seit 1826, deren Höhepunkt die Annahme der Carta von Bogotá im Jahre 1948 ist. Dies bedeutet das Ende der idealistischen Epoche des Panamerikanismus und zeigt den Beginn der konstitutionellen Periode an, mit anderen Worten, der neuen und entscheidenden Struktur des juristischen interamerikanischen Systems. Die Reihe der Verträge, Abkommen, Protokolle, Übereinkünfte usw., die zwischen den Staaten des Kontinents unterzeichnet wurden, rechtfertigt außerdem voll unsere Behauptung.

Der juristisch-politische Aufbau der amerikanischen Geschichte hat eine denkwürdige Tradition, die wir kurz beleuchten müssen, um die dargelegte Meinung besser verstehen $\mathrm{zu}$ könen. 


\section{Historische Grundlagen des amerikanischen juristischen Systems}

\section{a) Amerika, Vorläufer der Einheit des Kontinents}

Beleuchten wir kurz die Entwicklung der Normen des Internationalen Rechts in Amerika, insofern sie für unsere Schlußfolgerung von Bedeutung ist.

Stellen wir zunächst fest, daß die historische Tradition der juristischen Entwicklung in Amerika ebenso offenkundig wie einheitlich und homogen ist. Mit ihren Prinzipien hat sie nicht nur das Internationale Offentliche Universale Recht bereichert, sondern war auch berufen, in der eigenen Geschichte einen hervorragenden Platz einzunehmen.

So bemerken wir, daß die wiederholten Versuche universaler oder regionaler Zusammenschlüsse, die das Ziel hatten, für Interesse $\mathbf{z u}$ werben, den Frieden $\mathbf{z u}$ sichern und Gerechtigkeit bei internationalen Kontroversen zu üben, niemals Erfolg hatten, bis Amerika schließlich diese Wünsche zur aussichtsreichen Wirklichkeit machte. Es versteht sich, daß Gedanken von Weltorganisationen sich in verschwommener Form schon auf den ersten Seiten der Geschichte auffinden. So zum Beispiel ist der Amphiktyonische Rat des antiken Hellas die erste Institution, die auf der Basis universaler Solidarität funktionierte.

Im Mittelalter traten der Heilige Augustinus, der Heilige Thomas von Aquin, die Päpste Gregor VII. und Bonifatius VIII. öffentlich für eine Weltorganisation unter der Oberhoheit der Kirche ein. Georg Podiebrad, König von Böhmen, verlangte im Jahre 1492 die friedliche Konföderation der Staaten; der Franzose Pierre Dubois wollte die politisch-religiöse Konföderation; Campanella erstrebte eine internationale Gemeinschaft, die auf vertraglichen Bindungen beruhen sollte.

In der Neuzeit erdachte Heinrich IV. die Aufteilung Europas in 15 Staaten unter Jurisdiktion eines Generalrates; Eméric Crucé stellte sich in seiner „Le Nouveau Cinée" eine universale Union vor.

Später wollte der Landgraf von Hessen-Rheinfeld in „Der diskrete Katholik“ eine "Gesellschaft der Souveräne" mit einem Gerichtshof in Luzern. Der Quäker William Penn verlangte eine „Kammer oder ein europäisches Parlament“, und ein anderer Quäker, John Bellow, einen „europäischen Senat“. Der Abbé von Saint Pierre propagierte den ewigen Zusammenschluß der Herrscher, um den Frieden der Welt zu bewahren. Im Gegensatz dazu schlug Bentham vor, eine „Liga des gemeinsamen Nutzens" zu gründen, und Kant vertrat die Meinung, daß eine Föderation von republikanischen und freien Staaten eine Garantie für dauerhaften Frieden darstelle.

Unter den Amerikanern ist der Brasilianer Maía zu erwähnen; der Venezolaner General Francisco Miranda und der Nordamerikaner William Thornton, die sich eine große Staatenföderation vorstellten; aus Honduras José Cocilio del Valle, aus Argentinien Rozas und Monteagudo, der Chilene O'Higgins, der Uruguayer Gervasio Artigas, der Peruaner Juan Egaña und ganz besonders der große Befreier, General Simón Bolívar, der diese Ideen in die Praxis umsetzte, indem er in der Neuen Welt das Schauspiel einer internationalen Einigung vorführte. Nicht umsonst hat man gesagt, daß die Einheit der amerikanischen Republiken ein wirkliches, bemerkenswertes und einzigartiges Phänomen in der Geschichte der Menschheit darstellt. Das hängt damit zusammen, daß das Streben nach Einheit sich mit der eigenen Persönlichkeitsvorstellung des Amerikaners trifft; diese Persönlichkeit zeigt ihre Existenz seit jenen tragischen Tagen des Kampfes der spanischen Kolonien um ihre Unabhängigkeit. In Wahrheit entstand der Panamerikanismus aus dem Ideal und dem Kampf um die Freiheit, und keine Tradition kann denkwürdiger und ehrenvoller für ein Volk sein.

Zum Schluß müssen wir noch sagen, daß der Name Simón Bolívar einen abgöttischen Glanz unter den genannten Amerikanern erhielt, weil sein Einfluß auf die 
politisch-juristische Geschichte unseres Kontinents weltweite Wirkung hatte, da er ohne jeden Zweifel als der erste Vorläufer einer kontinentalen Solidarität und daher der Weltorganisation zu gelten hat.

\section{b) Der Kongreß von Panamá}

Nachdem Amerika seine Freiheit gewonnen hat, beginnt der Weg der großen Korrekturen. Denn von nun ab ist es Herr seiner selbst im Angesicht der grandiosen Bühne der Geschichte und benutzt nun eine völlig neue, für die damalige Welt fremde Sprache, denn es ist vom Glauben durchsetzt und proklamiert ihn mit heftiger Überzeugung.

Tatsächlich lernen Zivilisation und Geschichte eine neue universale Richtung kennen; die unerschütterliche Einheit wird dem ewigen und wahrhaftigen Wunsch der Menschheit moralische, materielle und geistige Konsistenz geben. Dieses Glaubensbekenntnis ist dank der Grundlagen, die der Befreier auf dem amphiktyonischen Kongreß von Panamá gelegt hat, welcher den Beginn der Geschichte des Panamerikanismus darstellt, für den Triumph bestimmt. In diesem Zusammenhang müssen wir daran erinnern, daß Bolívar in einem Brief an einen Engländer sagte: „Wir sind ein kleines Menschengeschlecht; wir besitzen eine abseitige Welt, weite Meere schneiden uns von außen ab; neu in fast allen Künsten und Wissenschaften, sind wir doch in gewisser Weise alt im Gebrauch der bürgerlichen Gesellschaft." Diese Überzeugung ist von Anfang an grundlegende und richtungsweisende Norm für Gedanken und Taten, die der besonderen Idee einer amerikanischen Welt folgen.

Im Einklang mit diesen Ideen versammelte sich der Kongreß von Panamá am 22. Juni 1826, und die Vertreter von Kolumbien, Zentralamerika, Perú und Mexiko schufen den historischen „Vertrag der ewigen Union, Liga und Konföderation“, der gemäß den zugehörigen Dokumenten und Protokollen alle Elemente enthielt, die den vergangenen Völkerbund charakterisierten. Weiter entspricht er grundsätzlich den Zielen der Konstitution der "magna civitas“, der Generalversammlung der Mitgliedstaaten; Wahrung des Friedens, Garantie der Unabhängigkeit und Unverletzlichkeit des Territoriums, international organisierte Rechtsprechung, Lösung von Konflikten mit friedlichen Mitteln, Versöhnung, Untersuchung und Schiedssprüche, Sanktionen gegenüber Angreifern, Kodifizierung des internationalen Rechtes, Verbot von Verträgen, die dem Assoziierungspakt entgegenstehen, Konsultationen zur Interpretation von Verträgen, Abschaffung von Seeräuberei und Kaperei, Universalität der Beziehungen usw. sind seine hauptsächlichen Ziele. Dieses Dokument ist der Vorläufer des Vertrages, der im Januar 1947 in Rio de Janeiro unterzeichnet wurde und unter dem Namen „Interamerikanischer Vertrag der gegenseitigen Hilfeleistung " bekannt ist.

So ist die Vorgeschichte des Panamerikanismus, der in ein Jahrhundert hineinwächst, das die Gründung eines Bundes der Nationen verwirklichen will, gemäß den Ideen, die zum erstenmal im Versailler Vertrag zum Ausdruck gebracht wurden.

\section{c) Nach dem Kongreß von Panamá}

Dem Kongreß von Panamá folgten 63 Jahre, die von Kriegen, Separationen, Mißtrauen und $\mathrm{Haß}$ gezeichnet sind. Dies war für die Einheit des Kontinents eine dunkle Epoche. Die Ereignisse von tiefgreifenden Folgen waren vor allem: die Auflösung Großkolumbiens mit den ihr vorangegangenen inneren Unruhen, die Auflösung der Zentralamerikanischen Republik mit den Kriegen zwischen den Staaten, aus denen sie gebildet war, die Invasion und Herrschaft Haitis in Santo Domingo, die Besetzung der Malwinen (Falkland-Inseln) durch die Engländer, der Krieg zwischen den Vereinigten Staaten und Mexiko, die europäischen Flottendemonstrationen gegenüber den amerikanischen Staaten, der Überfall und die 
Besetzung Nicaraguas durch William Walker und seine seeräuberischen Expeditionen, die britischen Intrigen in Mosquitia und den angrenzenden Gebieten, die Annexion von Santo Domingo durch Spanien, die Bombardierung von Buenos Aires durch die Engländer, die Eroberung von Buenos Aires und Montevideo und der Befreiungskampf, die Bombardierung von Callao und Valparaiso durch die Spanier, die militärische Aktion der Franzosen, Engländer und Spanier gegen Mexiko und die Errichtung der Monarchie unter dem Schutz des französischen Heeres; Bürgerkriege, Staatsstreiche, Tyranneien und Usurpationen in verschiedenen Republiken des Südens; der Bürgerkrieg zwischen den Nord- und Südstaaten von Amerika, der seinen Höhepunkt in der Ermordung des Präsidenten Lincoln fand; der Krieg Paraguays gegen die Dreierallianz (Argentinien, Brasilien, Uruguay), der Krieg Perús und Boliviens gegen Chile, der Krieg zwischen den zentralamerikanischen Nationen um die Wiederherstellung der Union usw.

Trotzdem finden sich in dieser wirren Zeit, die einige Schriftsteller die Zeit der Vorbereitungen nennen, vereinzelte Kräfte, die - von hohen Idealen durchdrungen - das friedliche Zusammenleben und die Sicherheit der amerikanischen Völker anstreben. In diesem Zusammenhang müssen wir die Konferenzen von Lima im Jahre 1847, von Santiago und Washington 1865, von Lima 1864 und Caracas 1883 erwähnen. Auf diesen Konferenzen haben die amerikanischen Republiken die Mittel vervollkommnet, mit denen sie eine Zusammenführung des weiten Gebietes ihrer Interessen erzielten. Dies geschah Schritt für Schritt, einmal mit Experimentieren, ein anderes Mal mit freiem Fortschritt und nicht selten rückläufig.

Bedenken wir schließlich, daß diese 63 Jahre, die auf den grundlegenden Kongreß von Panamá folgten, eine relativ kurze Zeitspanne sind, wenn man sich den ganzen langen Prozeß, der zur Verwirklichung gleicher Gedanken nötig war, vor Augen hält. Außerdem war diese Periode für Amerika ein Alarmzeichen in dem Sinne, daß es weniger bedroht von den Großmächten der Welt war, wenn seine Völker einig waren.

\section{d) Die interamerikanischen Konferenzen}

Mit dem Kongreß von Panamá beginnt eine Etappe eindeutigen Zusammenlebens zwischen den amerikanischen Staaten, das sich festigt auf zehn wichtigen Konferenzen, die das juristische amerikanische System endgültig schufen. Im folgenden zählen wir kurz ihre wichtigsten Beschlüsse auf:

1. Die erste internationale amerikanische Konferenz, die vom 2. Oktober 1889 bis zum 20. April 1890 in Washington tagte, ist die erste Phase einer echten Existenz der Nationen der Neuen Welt; trotzdem hielt man den Augenblick bereits für günstig, um die Grundlagen einer kontinentalen Organisation zu schaffen. Hier entstand eine Assoziation, die den Namen „Internationale Union der amerikanischen Republiken für die rasche Zusammenstellung und Verteilung kommerzieller Daten“ hatte, und es erfolgte die Gründung des „Handelsbüros der amerikanischen Republiken". Auch wurde eine Reihe von Resolutionen und Empfehlungen angenommen, von denen vor allem folgende zitiert werden müssen: Eine empfiehlt die Erstellung eines ständigen, allgemeinen und obligatorischen Schiedsgerichtsvertrages, um alle amerikanischen Konflikte zu lösen. Darin erkennt man die Tendenz der amerikanischen Länder, ihre Differenzen auf friedlichem Wege zu beseitigen. Das hatte übrigens einen gewichtigen Vorgänger, nämlich den Vertrag der Union, Liga und ständigen Föderation von Panamá. Hervorzuheben ist das Verbot des Rechtes auf Eroberungskriege, es verurteilt die Abtretung eines Teiles des Territoriums auf Grund von Gewalt und erklärt entsprechende Verträge für nichtig.

So organisierten sich die amerikanischen Republiken nach dieser Konferenz wie ein Bund der Nationen, und in der Sprache der Abmachungen und Verträge, mit 
denen sich unsere Organisation entwickelte, entstand etwas, was man schlicht "moralische Union“ zum Unterschied von einer politischen Union nannte.

2. Die zweite internationale amerikanische Konferenz, die vom 22. Oktober 1901 bis 31. Januar 1902 in Mexiko stattfand, reorganisierte das Handelsbüro und unterstellte es der Oberaufsicht eines Direktoriums, das sich aus den diplomatischen Repräsentanten aller amerikanischen Regierungen, die bei den Vereinigten Staaten von Amerika akkreditiert waren, und mit dem Staatssekretär dieser Nation als Präsidenten, zusammensetzte.

Das Schiedsgericht war Gegenstand neuer und intensiver Studien; es wurde ein Protokoll gefertigt, wonach die Mitglieder sich der Schiedsgerichtskonvention anschlossen, die in der ersten Friedenskonferenz von Den Haag 1899 unterzeichnet war, der nur die beiden amerikanischen Staaten, Mexiko und die USA, beigetreten waren.

3. Die dritte internationale amerikanische Konferenz versammelte sich in Rio de Janeiro vom 23. Juli bis zum 26. August 1906. Sie reorganisierte wiederum das Handelsbüro, sie behielt den Namen „Union“ bei und teilte ihr präzisere und detailliertere Aufgaben zu.

Bei dieser Gelegenheit gab der Staatssekretär der USA den Empfindungen der Regierung und des Volkes seines Landes mit folgenden bedeutsamen Worten Ausdruck: „Wir wünschen keine anderen Siege als die des Friedens, wir wollen kein fremdes Territorium, auch nicht mehr Souveränität als die Souveränität über uns selbst. Wir sind davon überzeugt, daß das kleinste und schwächste Mitglied der Völkerfamilie Anspruch auf Unabhängigkeit und auf Gleichheit der Rechte hat wie das größte Imperium; wir glauben, daß die Beachtung dieses Grundsatzes die entscheidende Sicherung des Schwachen gegenüber einem Angriff des Starken ist. Weder beanspruchen noch wünschen wir Rechte, Privilegien und Bevorzugungen, die wir nicht allen amerikanischen Republiken gewähren.“

Was in unserer Hemisphäre seit dieser historischen Deklaration schon Wirklichkeit war, mindestens im Bereich der Prinzipien, wurde erst 1945 der internationalen Gemeinschaft zuteil, als in der Charta der Vereinten Nationen die Gleichheit der Rechte von Mann und Frau und von großen und kleinen Nationen niedergelegt wurde.

4. Die vierte internationale amerikanische Konferenz kam vom 1. Juli bis 30. August 1910 in Buenos Aires zusammen. Bei dieser Gelegenheit beschlossen die versammelten Bevollmächtigten, den Namen des Organs der Konferenz, „Büro“, in „Panamerikanische Union“ umzubenennen, und übertrugen ihm größere Zuständigkeiten.

Es konstituierte sich eine „Union der Amerikanischen Nationen“, die mit zwei Administrationen arbeitete, eine in der Stadt Havanna, die andere in Rio de Janeiro, und die vollkommen aufeinander abgestimmt sind.

5. Die fünfte Konferenz, deren Tagungsort Santiago de Chile war, dauerte vom 25. März bis 23. Mai 1923; auch sie widmete sich diesen Organen und beschloß, daß die „Panamerikanische Union“ sich zu einem ständigen Organ konstitutierte. Die Grundlagen der Organisation wurden erweitert, und es wurde festgelegt, daß „die Vertretungen der Regierungen auf den Internationalen Amerikanischen Konferenzen im Rahmen der Panamerikanischen Union von eigenem Recht seien“. Auch durften die Regierungen zum erstenmal spezielle Vertreter vom Direktionsrat ernennen.

6. Die sechste Konferenz von Havanna im Jahre 1928 nahm eine Konvention über die Panamerikanische Union an, die aber nicht in Kraft treten konnte.

Die Verhandlungen dieser Konferenz dauerten vom 16. Januar bis 20. Februar 1928 und lagen also einundeinhalbes Jahr nach dem Kongreß, der in der Stadt Panamá zusammentrat, um die Hundertjahrfeier der berühmten Versammlung der Amerikanischen Nationen zu begehen, die von dem Befreier einberufen worden war. Von ihren Resolutionen verdienen folgende Punkte in Erinnerung gebracht zu werden: 
„Es wird der Beschluß anerkannt, eine Gesellschaft der amerikanischen Nationen zu konstituieren, die innerhalb der modernen Geltung des Internationalen Rechtes und auf der Grundlage der Rechtsgleichheit der Staaten den Idealen von Einheit und Gerechtigkeit entspricht, wie sie den Impuls zum Kongreß von Panamá gaben. Den Regierungen der amerikanischen Länder wird daher empfohlen, einen Kongreß von Bevollmächtigten einzuberufen, dessen ausschließliche Aufgabe die Erstellung des Paktes der genannten Gesellschaft sein soll."

Trotz des Wortlautes dieser Resolution nahm die Konferenz das Thema der Gesellschaft der amerikanischen Nationen nicht in ihr Programm auf: keine Regierung zeigte daran Interesse, da die amerikanischen Beziehungen weit davon entfernt waren, herzlich zu sein, und weil damals die Vereinigten Staaten in verschiedenen Republiken des Südens intervenierten und sie militärisch besetzt hielten.

Das ist der Grund dafür, daß die politische Vereinigung sowie das Projekt einer kontinentalen Liga keine Fortschritte im Leben unseres Kontinentes machten bis zum Zeitpunkt der siebten Konferenz. Trotzdem war diese Konferenz fruchtbar, und zwar für die Kodifizierung des Internationalen Privatrechtes, da die Konvention über das internationale Privatrecht oder der Code Bustamante eine der wichtigsten Etappen zur Kodifizierung dieses Zweiges der Jurisprudenz war.

Später, von Dezember 1928 bis Januar 1929, fand in der Stadt Washington eine Internationale Amerikanische Konferenz statt, deren Gegenstand Versöhnung und Schiedsgericht war. Hier wurden zwei Verträge angenommen, ein Generalschiedsgerichtsvertrag und ein Versöhnungsvertrag, zudem wurde ein Protokoll über das progressive Schiedsgericht festgelegt.

7. Die siebte Konferenz, an der drei Außenminister teilnahmen, fand vom 3. bis 26. Dezember 1933 in Montevideo statt. Sie beschloß die Bestätigung der unerschütterlichen Geltung friedlicher Mittel zur Lösung internationaler Konflikte; mit aller erforderlichen Kraft wurden sie angewandt, um den Frieden zwischen Paraguay und Bolivien wiederherzustellen. Diese Konferenz zählt zu den fruchtbarsten für Amerika, da sie viele Aspekte des Zusammenlebens der Völker berücksichtigte.

8. Im Jahre 1936 berief der Präsident Franklin D. Roosevelt die interamerikanische Konferenz zur Konsolidierung des Friedens nach Buenos Aires ein, auf der die „Deklaration über die Prinzipien der Solidarität und Inneramerikanischen Kooperation“ unterzeichnet wurde. Diese Deklaration enthält einige Grundsätze des Internationalen Offentlichen Amerikanischen Rechtes, weshalb wir einige ihrer Artikel im folgenden wiedergeben:

I. Die Nationen von Amerika proklamieren in Treue zu ihren republikanischen Institutionen die absolute Rechtsfreiheit, die uneingeschränkte Anerkennung ihrer Souveränität und die Existenz einer solidarischen Demokratie in Amerika;

II. Jede Aktion, die dazu beitragen liann, den Frieden in Amerika zu stören, gefährdet sie alle und jede einzelne und rechtfertigt die Anwendung von Konsultationen, wie es die Konvention zur Erhaltung, Sicherung und Wiederherstellung des Friedens vorsieht, die in dieser Konferenz unterzeichnet wurde;

III. Folgende Grundsätze sind von der internationalen amerikanischen Gemeinschaft angenommen worden:

a) Verbot territorialer Eroberungen und folglich Nicht-Anerkennung gewaltsamer Erwerbungen.

b) Verurteilung der Intervention eines Staates in die innen- oder außenpolitischen Angelegenheiten eines anderen Staates.

c) Verbot der Zwangseintreibung finanzieller Verpflichtungen.

d) Schlichtung aller Differenzen und Streitigkeiten zwischen den amerikanischen Nationen, gleich welchen Ursprungs oder welcher Art, auf dem Weg der Versöhnung, des Schiedsverfahrens oder der internationalen Gerechtigkeit.

Auf dieser Konferenz lieferten die Republiken Bolivien, Kolumbien, Kuba, Ekuador und die Dominikanische Republik verschiedene, aber sehr klare und direkte Diskussionsbeiträge zur Frage der Gründung eines Bundes der amerikanischen 
Nationen, während El Salvador und Guatemala einen „Generalvertrag der Solidarität und Kooperation“ vorschlugen, und Haiti eine "Internationale Amerikanische Union" gründen wollte, und zwar auf der Grundlage der gegenseitigen Garantie der politischen Unabhängigkeit und der territorialen Integrität der Staaten des Kontinents.

Es kann als feststehend gelten, daß auf dieser Konferenz das Prinzip der kontinentalen Solidarität begann, Formen anzunehmen, die kurze Zeit darauf mit aller Schärfe umrissen werden sollten. So bekam die Konferenz trotz ihres speziellen Charakters ebenso großes oder mehr Gewicht als irgendeine der vorhergehenden. 9. Die neunte Konferenz fand vom 9. bis 27. Dezember 1938 in Lima statt und billigte die Deklaration der amerikanischen Grundsätze mit folgenden Worten:

a) Die Einmischung eines Staates in die inneren und äußeren Angelegenheiten eines anderen ist unzulässig;

b) Alle Streitigkeiten mit internationalem Charakter müssen auf friedlichem Wege beigelegt werden;

c) Die Anwendung von Gewalt als Instrument der nationalen oder internationalen Politik ist verboten;

d) Die Beziehungen zwischen den Staaten müssen den Normen des internationalen Rechts entsprechen;

e) Die Achtung und gewissenhafte Wahrung der Verträge sind unabdingbare Normen für die friedliche Entwicklung der Beziehungen zwischen den Staaten und können nur bei gegenseitiger Zustimmung. abgeändert werden;

f) Die friedliche Zusammenarbeit $\mathbf{z w i s c h e n ~ d e n ~ R e p r a ̈ s e n t a n t e n ~ d e r ~ v e r s c h i e d e n e n ~}$ Staaten und die Entwicklung des Gedankenaustausches zwischen ihren Völkern tragen zum besseren Verständnis der Probleme jedes einzelnen und der Gemeinschaft bei und erleichtern gleichzeitig die friedliche Lösung internationaler Kontroversen;

g) Der wirtschaftliche Wiederaufbau kommt dem nationalen und dem internationalen Wohlstand zugute sowie dem Frieden zwischen den Völkern; er dient der Aufrechterhaltung der entwickelten Grundsätze.

10. $\mathrm{Zu}$ Beginn des Jahres 1945 stellten sich wichtige Fakten ein, die im Zusammenhang mit dem Verlauf des Zweiten Weltkrieges standen, und es wurde notwendig, einen Richtungswechsel, eine Neuorientierung vorzunehmen, und so begann eine neue Phase der Entwicklung des Interamerikanischen Systems. Aus diesem Grunde versammelten sich wiederum unsere Bevollmächtigten in der Stadt Mexiko vom 21. Februar bis 8. März 1945 auf der Interamerikanischen Konferenz über die Probleme des Krieges und des Friedens. Das allgemeine Ergebnis dieser Konferenz war sehr entscheidend. Es ging noch hinaus über die Beschlüsse zur gegenseitigen Hilfe und amerikanischen Solidarität, die in der Akta von Chapultepec niedergelegt sind, und die mit den entsprechenden Reorganisationsbeschlüssen das Interamerikanische System konsolidierte.

Zum Schluß unserer Ausführungen ist die sogenannnte „Deklaration von Mexiko“ von Interesse, die folgende Grundsätze aufstellte:

a) Das Internationale Recht ist Verhaltensnorm für alle Staaten.

b) Die Staaten sind vor dem Rechte gleich.

c) Jeder Staat ist frei und souverän. Kein Staat kann sich in die inneren oder äußeren Angelegenheiten eines anderen einmischen.

d) Das Territorium der amerikanischen Staaten ist unverletzlich und unveränderlich, ausgenommen im Falle friedlicher Vereinbarungen.

e) Die amerikanischen Staaten erkennen territoriale Eroberungen nicht an.

f) Aufgabe der amerikanischen Staaten ist die Wahrung des Friedens und Aufrechterhaltung der bestmöglichen Beziehungen zu allen Staaten.

g) Konflikte zwischen den Staaten dürfen nur auf friedlichem Weg gelöst werden.

h) Angriffskriege jeder Art sind verboten. 
i) Ein Angriff auf einen amerikanischen Staat bedeutet Angriff auf alle amerikanischen Staaten.

j) Die amerikanischen Staaten erklären sich im Hinblick auf ihre Ziele und gemeinsamen Interessen solidarisch.

k) Die amerikanischen Staaten wiederholen ihr glühendes Bekenntnis zu den demokratischen Prinzipien, die sie als wesentlich für den Frieden ansehen.

1) Das Ziel des Staates ist die Glückseligkeit des Menschen innerhalb der Gesellschaft. Die Interessen der Gemeinschaft müssen mit den Rechten des einzelnen harmonisieren. Der amerikanische Mensch kann ohne Gerechtigkeit nicht leben. Ebensowenig duldet er ein Leben ohne Freiheit.

m) An erster Stelle steht unter den Menschenrechten die Gleichheit der Möglichheiten, an allen geistigen und materiellen Gütern teilzuhaben, die unsere Zivilisation durch erlaubte Ausübung von Aktivität, Fleiß und Erfindungsgeist bietet.

n) Bildung und materieller Wohlstand sind für den Fortschritt der Demokratie unabdingbar.

o) Die wirtschaftliche Zusammenarbeit ist entscheidend für den allgemeinen Wohlstand der amerikanischen Nationen. Das Elend jedes ihrer Völker wie Armut, Unterernährung und Mangel an Hygiene belastet jede Nation und folglich alle gemeinsam.

p) Die amerikanischen Staaten betrachten die gerechte Koordinierung aller ihrer Interessen als unerläßlich zur Schaffung einer reichen Wirtschaft, in der Natur und menschliche Arbeit zusammenwirken, um die Lebensbedingungen aller Völker des Kontinents zu heben.

q) Die amerikanische Gemeinschaft dient den Idealen der Zusammenarbeit der Welt.

11. Die neunte Konferenz fand im Jahre 1948 in Bogotá statt, und man kann sagen, daß sie die wichtigste von allen war, da auf ihr die Charta der Organisation der Amerikanischen Staaten und andere wichtige Maßnahmen wirtschaftlicher, kultureller und rechtlicher Art gebilligt wurden. Hierbei muß der amerikanische Vertrag zu friedlichen Lösungen (Pakt von Bogotá) erwähnt werden, der die geltenden Rechtsformen zur friedlichen Lösung der Konflikte koordiniert und konsolidiert und einige neue hinzufügte.

Die Charta von Bogotá zählt in Artikel 5 die amerikanischen Prinzipien auf und lautet wie folgt:

„Die amerikanischen Staaten bestätigen erneut folgende Prinzipien:

a) Die internationale Ordnung basiert vor allem auf dem Respekt vor der Persönlichkeit, der Souveränität und Unabhängigkeit der Staaten und auf der treuen Erfüllung der in den Verträgen des internationalen Rechtes festgelegten Verpflichtungen.

b) Das internationale Recht verpflichtet die Staaten in ihren gegenseitigen Beziehungen.

c) Der gute Glaube muß die Beziehungen zwischen den Staaten beherrschen.

d) Die Solidarität der amerikanischen Staaten und die hohen Ziele, die sie sich gestellt haben, erfordern ihre politische Organisation zur effektiven Ausübung der repräsentativen Demokratie.

e) Die amerikanischen Staaten verurteilen den Angriffskrieg; der Sieg schafft keine Rechte.

f) Der Angriff auf einen amerikanischen Staat ist gleichbedeutend mit dem Angriff auf alle anderen amerikanischen Staaten.

g) Kontroversen mit internationalem Charakter, die zwischen zwei oder mehreren amerikanischen Staaten auftreten, müssen auf friedlichem Weg geschlichtet werden.

h) Die wirtschaftliche Zusammenarbeit ist Grundlage für den Wohlstand und Reichtum der Völker des Kontinents. 
i) Gerechtigkeit und soziale Sicherheit sind Grundlagen für einen dauerhaften Frieden.

j) Die amerikanischen Staaten proklamieren die fundamentalen Grundrechte der Person ohne Diskriminierung der Rasse, der Nationalität, des Glaubens oder Geschlechtes.

k) Die geistige Einheit des Kontinents basiert auf der Achtung der kulturellen Eigenart der amerikanischen Länder und erfordert ihre enge Zusammenarbeit zur Erreichung der hohen Ziele der menschlichen Kultur.

1) Die Bildung der Völker muß auf die Grundsätze der Gerechtigkeit, der Freiheit und des Friedens ausgerichtet sein.

Auf dieser Konferenz wurde beschlossen, daß die beratende Versammlung der Außenminister bei Auftreten von Problemen dringenden Charakters und von allgemeinem Interesse für die amerikanischen Staaten zusammentreten müsse und daß sie als Konsultationsorgan fungieren solle.

Die beratende Versammlung wurde 1936 auf der interamerikanischen Konferenz des Friedens in Buenos Aires gegründet; 1938 erhielt sie auf der achten Konferenz zu Lima ihre definitive Gestalt und trat bei elf Gelegenheiten in Aktion: 1939 in Panamá, 1940 in Havanna, 1942 in Rio de Janeiro, 1951 in Washington, 1959 in Chile, 1960 in Costa Rica, 1962 in Montevideo und 1967 in Washington und Buenos Aires.

Das Konsultativverfahren, das im Vertrag der gegenseitigen Hilfe niedergelegt und in die Charta für Fälle von bewaffneten Angriffen und Bedrohungen des Friedens aufgenommen wurde, ist zweimal angewandt worden; in beiden Fällen wurde auf Artikel 6 des Vertrages von Rio de Janeiro zurückgegriffen.

Es muß noch gesagt werden, daß der Name „Union der amerikanischen Republiken“ oder des früheren „Interamerikanischen Systems" durch die Bezeichnung „Organisation der Amerikanischen Staaten (OAS)" ersetzt wurde.

So machten die Internationalen Konferenzen, die 1890 begonnen haben, einen Bezeichnungswechsel durch und heißen seit 1948 „Die Interamerikanische Konferenz". Diese ist innerhalb der Struktur der Charta höchstes Organ und das einzige, das in Fragen der allgemeinen Politik der Organisation tätig werden kann.

12. Als letzte fand am 1. März 1954 in Caracas die zehnte internationale amerikanische Konferenz statt. Zu den Verhandlungspunkten zählte die Schaffung des Interamerikanischen Gerichtshofes, der schon Thema verschiedener anderer Konferenzen war, nämlich der fünften von Santiago, der sechsten von Havanna, der Interamerikanischen Konferenz zur Konsolidierung des Friedens von Buenos Aires und der siebten Interamerikanischen Konferenz von Montevideo.

13. Anstelle der elften Interamerikanischen Konferenz, die 1959 in Quito (Ekuador) hätte stattfinden sollen, aufgrund der Streitigkeiten zwischen Perú und Ekuador jedoch auf unbestimmte Zeit verschoben wurde, wurde die dritte Interamerikanische Außerordentliche Konferenz nach Buenos Aires im Februar 1967 einberufen.

Auf dieser Konferenz wurde die Charta der OAS modifiziert und durch den Zusatz der sozialen wirtschaftlichen Solidarität gegenüber regionalen Verpflichtungen der politischen und militärischen Solidarität modernisiert.

So entstand mit dem "Protokoll von Buenos Aires" also eine modernisierte, fähige und mit neuer Kraft erfüllte OAS. Die Prinzipien der wirtschaftlichen und sozialen Solidarität wurden auf der Konferenz von Rio de Janeiro ausgearbeitet, revidiert in Panamá und endgültig formuliert vom Rat in Washington und blieben so dem System einbezogen.

Das höchste oberste Organ des Systems, die oben erwähnte „Interamerikanische Konferenz", war 13 Jahre hindurch außer Funktion. Sie wurde erst durch außerordentliche Konferenzen, dann durch die Versammlung der Außenminister ersetzt, die einmal im Jahr zusammentritt und als Forum für Diskussionen über regionale Probleme dienen sollte. 
Anstatt einer einzigen politischen Organisation, dem Ständigen Rat der OAS, existieren jetzt drei Räte gleicher Hierarchie:

a) der Rat der OAS;

b) der interamerikanische wirtschąftliche und soziale Rat, dessen Exekutivkomitee das interamerikanische Komitee der Alilianz für den Fortschritt ist. Seine Aufgabe ist, die allgemeinen Ziele der Allianz in die Struktur der OAS einzubauen;

c) der Interamerikanische Rat für Bildung, Wissenschaft und Kultur.

Aus den bisherigen Ausführungen ergeben sich folgende Schlußfolgerungen:

1. Unmißverständlich zeichnen sich die Prinzipien ab, die dem Internationalen Amerikanischen Offentlichen Recht allgemein anerkannte Wirklichkeit verleihen:

a) Amerikanische Auffassung von der Staatssouveränität

b) Amerikanische Auffassung von der Rechtsgleichheit der Staaten

c) Nicht-Intervention

d) Illegalität der Zwangseinziehung öffentlicher Schulden

e) Friedliche Lösung internationaler Kontroversen

f) Kollektive Sicherheit der amerikanischen Staaten

g) Gleichheit von Staatsbürger und Ausländer

h) Nicht-Verantwortung des Staates im Falle von Schäden, die Ausländer durch Nicht-Verschulden des Staates erlitten haben

i) Anerkennung der De-facto-Regierungen

j) Uti possidetis iuris

2. Mit der Annahme der Charta der OAS wurde eine feste und beständige Grundlage für das amerikanische Rechtssystem erreicht und

3. es fehlt zur Vervollständigung ihrer juristischen Struktur der Interamerikanische Rechtshof, der es möglich machen würde, die amerikanische Welt als die anzusehen, der am meisten Vollkommenheit, Einigkeit und Reife innewohnt, ein Ideal, das in der amerikanischen Seele seit den unsterblichen Zeiten Simón Bolívars verwurzelt ist. 